\title{
Thermoluminescence responses of photon- and electron-irradiated lithium potassium borate co-doped with $\mathrm{Cu}+\mathrm{Mg}$ or $\mathrm{Ti}+\mathrm{Mg}$
}

\begin{abstract}
New glasses Li2CO3-K2CO3-H3BO3 (LKB) co-doped with $\mathrm{CuO}$ and $\mathrm{MgO}$, or with $\mathrm{TiO} 2$ and $\mathrm{MgO}$, were synthesized by the chemical quenching technique. The thermoluminescence (TL) responses of $\mathrm{LKB}: \mathrm{Cu}, \mathrm{Mg}$ and $\mathrm{LKB}: \mathrm{Ti}, \mathrm{Mg}$ irradiated with $6 \mathrm{MV}$ photons or $6 \mathrm{MeV}$ electrons were compared in the dose range 0.5-4.0 Gy. The standard commercial dosimeter LiF:Mg,Ti (TLD-100) was used to calibrate the TL reader and as a reference in comparison of the TL properties of the new materials. The dependence of the responses of the new materials on 60Co dose is linear in the range of 1-1000 Gy. The TL yields of both of the codoped glasses and TLD-100 are greater for electron irradiation than for photon irradiation. The TL sensitivity of LKB:Ti, Mg is 1.3 times higher than the sensitivity of LKB:Cu, Mg and 12 times less than the sensitivity of TLD-100. The new TL dosimetric materials have low effective atomic numbers, good linearity of the dose responses, excellent signal reproducibility, and a simple glow curve structure. This combination of properties makes them suitable for radiation dosimetry.
\end{abstract}

Keyword: Thermoluminescence; Dosimetry; Lithium potassium borate; Copper; Titanium; Magnesium dopants 